\title{
NATURAL ALKALOID SECURININE IS A PROMISING SCAFFOLD FOR THE DEVELOPMENT OF NEUROPROTECTIVE DRUGS
}

\author{
S.C. Klochkov' ${ }^{1}$, Yu.R. Aleksandrova', A.V. Semakov', M.E. Neganova'
}

${ }^{1}$ IPAC RAS, 142432, Russia, Chernogolovka, Severny pr., 1.

DOI: 10.19163/MedChemRussia2021-2021-157

E-mail:klochkov@ipac.ac.ru

Securinine, a natural alkaloid from the roots of the genus Securinega, is a compound with a unique structure and has a wide spectrum of action, including anti-inflammatory, antibacterial, neuroprotective and antitumor. It has several reactive sites and is an interesting scaffold for the synthesis of biologically active compounds. Securinine is a competitive antagonist of the GABAA receptor, potentiating learning and memory [1], and can be considered as a promising basis for the synthesis of compounds with neuroprotective activity.

We have obtained a wide range of adducts of the alkaloid securinine with pharmacophoric amines, and based on the results of their in vitro study of biological activity, we have identified the leader compound allomargaritarine with a pronounced neuroprotective effect due to its influence on several key links in the development of the pathological process of neurodegenerative diseases. In addition to antioxidant and mitoprotective properties [2-3], allomargaritarine has been shown to be able to prevent the formation of pathological forms of $A \beta$ by inhibiting the activity of the BACE1 enzyme, as well as the ability to prevent aggregation of $A \beta 1-42$. In an in vivo series of experiments using a model of scopolamine-induced amnesia for allomargaritarine, it was shown that impairments in learning and the formation of episodic and spatial memory in mice associated with dysfunction of acetylcholine neurotransmission were eliminated. A similar effect was found in the study of both short-term and longterm memory of transgenic 5xFAD mice, the pathological phenotype of which includes the disorders observed in Alzheimer's disease. Subsequent analysis of $A \beta$ content in animal brain slices revealed a significant decrease in the number of amyloid inclusions in transgenic mice treated with allomargaritarine.

Thus, derivatives of the unique natural indolizidine alkaloid securinine, first of all, allomargaritarine, can act as a promising basis for the creation of therapeutic agents with a multipharmacological type of neuroprotective activity.

\section{References}

[1] X. Lin, W. Guolin and Z. Jun-tian, Planta Med. 2002, 68 (8), 752-3

[2] M.E. Neganova, S.G. Klochkov et al., CNS Neurol. Disord. Drug Targets, 2016, 15 (1), 102-107

[3] M.E. Neganova, S.G. Klochkov et al., CNS Neurol Disord Drug Targets. 2017;16 (3), 351-355 